Delgado-Martín, J.L.; Garoz-Puerta, I.; Miguel-Tobal, F. y Martínez-de-Haro, V. (2020). Anthropometry and Strength, Its Influence on the Sit And Reach Test. Revista Internacional de Medicina y Ciencias de la Actividad Física y el Deporte vol. 20 (78) pp. 369-380 Http://cdeporte.rediris.es/revista/revista78/artantropometria1157.htm

DOI: http://doi.org/10.15366/rimcafd2020.78.012

\title{
ORIGINAL
}

\section{ANTROPOMETRÍA Y FUERZA, SU INFLUENCIA SOBRE EL TEST SIT AND REACH}

\section{ANTHROPOMETRY AND STRENGTH, ITS INFLUENCE ON THE SIT AND REACH TEST}

\author{
Delgado-Martín, J.L. ${ }^{1}$; Garoz-Puerta, I. ${ }^{1}$; Miguel-Tobal, F. ${ }^{2}$ y Martínez-de- \\ Haro, V. ${ }^{1}$ \\ ${ }^{1}$ Departamento de Educación Física, Deporte y Motricidad Humana. Universidad Autónoma de \\ Madrid (España) jose.delgado@uam.es, nacho.garoz@uam.es, vicente.martinez@uam.es \\ ${ }^{2}$ Escuela de Medicina de la Educación Física y el Deporte. Facultad de Medicina. Universidad \\ Complutense de Madrid (España) miguelto@ucm.es
}

Código UNESCO / UNESCO code: 3212 Salud Pública / Public Health Clasificación Consejo de Europa / Council of Europe Classification: 17 Otros (Actividad Física y Salud) / Others (Physical Activity and Health)

Recibido 2 julio de 2018 Received July 2, 2018

Aceptado 1 de agosto de 2018 Accepted August 1, 2018

\section{RESUMEN}

Se analiza la influencia que tienen las variables cineantropométricas y dinamométricas sobre el test Sit and Reach en deportistas amateur. Se tomaron veinte variables antropométricas, cuatro pruebas de dinamometría, el test Sit and Reach y la actividad física realizada. Encontramos diferencias significativas en los valores de flexibilidad entre hombres y mujeres. Las ecuaciones de predicción fueron diferentes en hombres y en mujeres $(30.6 \%$ y $32.5 \%)$, en las mujeres apareció la talla como factor influyente. La ecuación general de predicción calculada se obtiene un $39,1 \%$ (moderada). Hombres y mujeres se diferencian significativamente en todas las variables antropométricas excepto en pliegue subescapular y suprailiaco. ¿Qué otros factores influirían sobre una posible predicción del resultado? Los valores de flexibilidad no se consideran influenciados por las variables antropométricas, dinamométricas y la tipología de actividad física realizada. El índice de fuerza no correlaciona con el valor del test Sit and Reach.

PALABRAS CLAVE: condición física relacionada con la salud, antropometría, pruebas de flexibilidad, dinamómetro, pruebas de fuerza. 


\begin{abstract}
Analyzes the influence of kinanthropometric and dynamometric variables on the Sit and Reach test in amateur athletes. We used twenty anthropometric variables, four dynamometry tests, the Sit and Reach test and the amount of physical activity practiced. Significant differences were found between men and women in terms of flexibility levels. The prediction equations were differed according to gender (30.6\% men and $32.5 \%$ women), height being an influential factor in women. The general prediction equation calculated, the level is $39.1 \%$ (moderate). Men and women show significant differences in all anthropometric variables except subscapular and suprailiac skinfolds. What other factors influence the kind of results that can be expected? The results of the flexibility test are not influenced by the anthropometric and dynamometric variables, or by the type of physical activity performed. The strength index does not correlate with the Sit and Reach test value.
\end{abstract}

KEYWORDS: Physical fitness related health, Anthropometry, flexibility testing, dynamometer, strength testing.

\title{
INTRODUCCIÓN
}

Desde la segunda mitad del siglo XX se prestó un interés específico a la relación entre actividad física y condición física con respecto a la consideración sobre el estado de salud y la capacidad funcional (1). Llegando a establecerse que tres de los componentes de la condición física para la salud son la flexibilidad, la fuerza y la composición corporal, componentes mejorables con la práctica de actividad física adecuada y asociados a un bajo riesgo en el desarrollo de enfermedades derivadas del sedentarismo (2).

Así el test Sit and Reach (SRT) clásico, cuyo diseño original pertenece a Wells \& Dillon (3), está incluido como parte de las baterías de pruebas por diferentes estamentos cualificados como la American Alliance for Health Physical Education, Recreation \& Dance (4), por el Consejo de Europa (5), por el Australian Council for Health, Physical Education and Recreation (6) o por la Canadian Physical Activity, Fitness and Lifestyle Appraisal (7). La interpretación que se le concede a dicho test sobre los resultados es que la mayor distancia alcanzada deriva en mayor grado de flexibilidad lumbar, pélvica y de la musculatura isquiosural (3).

Aunque nos encontramos con un test que ha sido ampliamente justificado y utilizado, muchos estudios lo cuestionan al determinar qué hay factores anatómicos que pueden alterar sus resultados como pudieran ser la posición de la cabeza (8); antepulsión y abducción escapulo-humeral, flexibilidad en columna vertebral y articulaciones intervertebrales (9); longitud de miembros en extremidades superiores e inferiores (10); posición de los tobillos y de los dedos de los pies (11); intervención de pelvis y cadera (12); compensación de los grupos musculares involucrados (13). 
Otros estudios determinan que la validez, fiabilidad y criterio del SRT tiene moderada estimación en la medida de la flexibilidad isquiosural y baja en la flexibilidad lumbar $(12,14-16)$, determinan diferencias por sexos y/o grupos de edad $(17,18)$, establecen una necesidad en el estudio de muestras con deportistas y población físicamente activa (19).

Una de las técnicas más utilizadas para el cálculo de la composición corporal es la cineantropometría. Se encarga de medir las dimensiones corporales como: peso, talla, cintura, cadera, diámetros, perímetros y pliegues cutáneos. Conforme a la Organización Mundial de la Salud (OMS): "los índices antropométricos son combinaciones de mediciones y resultan esenciales para la interpretación de éstas: es evidente que un valor por sí solo no tiene significado a menos que esté relacionado con alguna variable o indicador" (20, p.8).

La American College of Sports Medicine (ACSM, 21) reconoce que la fuerza muscular es una cualidad física necesaria para la salud. Reiterando que ante su desarrollo y mantenimiento nos encontramos con una condición necesaria para conservar las habilidades funcionales, permitir la ejecución de las actividades diarias y de utilidad en la prevención y rehabilitación de patologías musculoesqueléticas.

En las relaciones establecidas entre la dinamometría, la flexibilidad y la antropometría nos encontramos que: Chandrasekaran et al., (22) determinaron que la talla, edad y peso son importantes predictores de la fuerza; Silva et al., (23) indicaron una influencia de algunas variables antropométricas y la edad con la dinamometría, también como el exceso de peso provoca una limitación en la flexibilidad; Sharma \& Kailashiya (24) mostraron correlación significativa de la talla y composición corporal con dinamometría y flexibilidad.

El objetivo de nuestra investigación es analizar las relaciones existentes entre las variables cineantropométricas y dinamométricas con el SRT en una muestra de personas que practican actividad físico deportiva (AFD) a nivel recreacional.

\section{MATERIAL Y MÉTODOS}

La muestra está compuesta por un total de 491 personas de ambos sexos (289 hombres y 202 mujeres) que practican AFD, de carácter recreacional, con un año mínimo de antigüedad en la práctica y siguiendo elementos recogidos que corresponderían a los patrones establecidos como saludables por la ACSM (31); con edades comprendidas entre 18 y 68 años y una edad media de 36 años (36.2 \pm 12.8$)$ en hombres y de 34 años (33.6 \pm 12.7$)$ en mujeres. Acudieron a la Escuela de Medicina de la Educación Física y del Deporte de la Universidad Complutense de Madrid (UCM) para la realización de una evaluación médicodeportiva. Todos los participantes exponen no tener antecedentes recientes de patologías, no tomar medicación y no practicar deporte a nivel de competición. Los participantes recibieron información sobre las medidas a realizar en el estudio y dieron su consentimiento por escrito. Los datos que se recogieron se codificaron para respetar la confidencialidad. Esta investigación fue aprobada 
por el Comité de Ética para las investigaciones relacionadas con seres humanos de la Universidad Autónoma de Madrid (UAM), CEI-90-1674.

Fueron seleccionadas como criterio de inclusión en la selección muestral aquellas historias clínicas que tuvieran los siguientes indicadores: sexo, edad, peso, talla, cintura, cadera, ICC, diámetros biestiloideo, biepicondilar y bicondíleo, perímetros brazo contraído y de pierna máxima, pliegues del tríceps, subescapular, suprailiaco, abdominal, muslo anterior, pierna medial, dinamometría de mano derecha, mano izquierda, espalda, y de piernas, flexibilidad y actividad física realizada.

El diseño estadístico realizado, ha sido no experimental basado en metodología de carácter descriptivo, retrospectivo, observacional y de prevalencia.

Todas las mediciones se realizaron en el laboratorio de la Escuela de Medicina del Deporte de la UCM, sin realizar ningún tipo de actividad física previa a la toma de mediciones. Se les realizó una anamnesis médico-deportiva y otras variables relacionadas, como la práctica, tipo, duración y frecuencia de las actividades físicas se registraron mediante cuestionario propio.

Las valoraciones cineantropométricas fueron realizadas por médicos deportivos de la Escuela acreditados por la International Society for the Advancement of Kinanthropometry (ISAK) de acuerdo con las técnicas estandarizadas y mediante certificado de la ISAK (26).

Los instrumentos de medida utilizados fueron para la talla un tallímetro Holtain®, para la medición del peso fue utilizada una báscula Lafayette® modelo Detecto, para la medición de los pliegues se utilizó un plicómetro Holtain®, los diámetros fueron medidos con un paquímetro o antropómetro Holtain ${ }^{\circledR}$, los perímetros fueron medidos mediante cinta antropométrica flexible. La medición de la dinamometría se realizó a través de un dinamómetro manual digital modelo TKK5401 Tecsymp® y dinamómetro de espalda y piernas modelo TKK-5002 Tecsympß.

El protocolo de flexibilidad (SRT) y dinamometría se realizó siguiendo recomendaciones propuestas por la AAHPERD (4), utilizando un cajón estándar marca Lafayette® y situando como marca valor cero a $23 \mathrm{~cm}$ antes del apoyo de los pies.

El análisis estadístico se realizó con el programa Statistical Package for Social Sciences (SPSS 23)®. Se obtuvieron los datos para describir estadísticamente la muestra, datos de centralización, dispersión y de forma (media aritmética, desviación estándar, varianza, asimetría y curtosis). El nivel de significación elegido fue $<.05$. Para la interpretación de los resultados obtenidos se utilizaron los valores establecidos por Hopkins (27): trivial $(<.1)$, small $(r \leq .3)$, moderate $(r$ $\geq .3<.5)$, large $(r \geq .5<.7)$, very large $(r<.9)$, almost perfect $(r>.9)$. Se realizó una prueba $t$ de muestras independientes previa medida de la normalidad. Para evaluar la asociación entre las diferentes variables, se calcularon mediante una correlación de Pearson y se determinó su significación estadística. Con el 
propósito de identificar las asociaciones independientes entre las diversas variables se realizó un análisis de regresión lineal por pasos y se calculó el coeficiente de determinación $\mathrm{R}^{2}$. El análisis permitió calcular la significación de las variables y determinar cuáles de ellas tienen influencia sobre la flexibilidad.

\section{RESULTADOS}

Entre los resultados descriptivos mostramos en la tabla 1 las medias aritméticas y desviaciones estándar por décadas de edad y sexo, de la flexibilidad $(\mathrm{cm})$, medida a través del SRT.

Tabla 1: Estadística descriptiva valores de flexibilidad.

\begin{tabular}{lcccc}
\hline & & Hombres( media \pm DS) & & Mujeres( media $\pm D S$ ) \\
\hline $\mathbf{1 8 / 1 9}$ & $\mathrm{N}=28$ & $30.48 \pm 8.10$ & $\mathrm{~N}=24$ & $30.19 \pm 13.72$ \\
$\mathbf{2 0 / 2 9}$ & $\mathrm{N}=76$ & $28.63 \pm 10.16$ & $\mathrm{~N}=84$ & $30.87 \pm 7.92$ \\
$\mathbf{3 0 / 3 9}$ & $\mathrm{N}=63$ & $29.40 \pm 8.72$ & $\mathrm{~N}=37$ & $35.27 \pm 8.19$ \\
$\mathbf{4 0 / 4 9}$ & $\mathrm{N}=78$ & $25.63 \pm 7.57$ & $\mathrm{~N}=23$ & $32.57 \pm 10.55$ \\
$\mathbf{5 0 / 5 9}$ & $\mathrm{N}=34$ & $23.60 \pm 6.54$ & $\mathrm{~N}=30$ & $33.03 \pm 6.35$ \\
$\mathbf{6 0 / 6 8}$ & $\mathrm{N}=9$ & $28.90 \pm 11.84$ & $\mathrm{~N}=4$ & \\
\hline
\end{tabular}

Procedemos a expresar en la tabla 2 las medias aritméticas y desviaciones estándar por década de edad y sexo, medida realizada sobre la prueba de dinamometría.

Tabla 2: Estadística descriptiva valores de dinamometría.

\begin{tabular}{|c|c|c|c|c|c|c|c|c|}
\hline & & $\begin{array}{c}\text { Total } \\
H(n=289) \\
M(n=202)\end{array}$ & $\begin{array}{c}18 / 19 \\
H(n=28) \\
M(n=24)\end{array}$ & $\begin{array}{c}20 / 29 \\
H(n=76) \\
M(n=84)\end{array}$ & $\begin{array}{c}30 / 39 \\
H(n=63) \\
M(n=37)\end{array}$ & $\begin{array}{c}40 / 49 \\
H(n=78) \\
M(n=23)\end{array}$ & $\begin{array}{c}50 / 59 \\
H(n=34) \\
M(n=30)\end{array}$ & $\begin{array}{c}60 / 68 \\
H(n=9) \\
M(n=4)\end{array}$ \\
\hline & & media $\pm \mathrm{DS}$ & media $\pm D S$ & media $\pm D S$ & media $\pm D S$ & media $\pm D S$ & media $\pm D S$ & media $\pm D S$ \\
\hline \multirow[b]{2}{*}{ 只 } & $\mathrm{H}$ & $42.05 \pm 7.00$ & $40.77 \pm 5.70$ & $41.52 \pm 7.11$ & $42.78 \pm 7.93$ & $44.10 \pm 7.03$ & $41.41 \pm 6.92$ & $36.10 \pm 8.53$ \\
\hline & $\begin{array}{l}M \\
T\end{array}$ & $\begin{array}{l}26.96 \pm 4.63 \\
36.36 \pm 9.60\end{array}$ & $\begin{array}{l}28.17 \pm 5.08 \\
35.37 \pm 8.29\end{array}$ & $\begin{array}{l}27.70 \pm 4.37 \\
34.90 \pm 9.12\end{array}$ & $\begin{array}{c}26.10 \pm 4.56 \\
37.50 \pm 10.49\end{array}$ & $\begin{array}{c}25.87 \pm 5.62 \\
38.60 \pm 10.70\end{array}$ & $\begin{array}{l}25.94 \pm 2.60 \\
36.25 \pm 9.40\end{array}$ & $34.30 \pm 8.81$ \\
\hline \multirow{2}{*}{$\bar{\Sigma}$} & $\begin{array}{l}\mathbf{H} \\
\mathbf{M}\end{array}$ & $\begin{array}{l}40.29 \pm 7.08 \\
26.07+4.39\end{array}$ & $\begin{array}{l}38.01 \pm 6.68 \\
27.18+3.77\end{array}$ & $\begin{array}{l}40.53 \pm 7.10 \\
26.01+4.15\end{array}$ & $\begin{array}{l}40.89 \pm 7.84 \\
26.06+4.49\end{array}$ & $\begin{array}{l}42.20 \pm 7.52 \\
25.93 \pm 5.68\end{array}$ & $\begin{array}{l}38.49 \pm 6.34 \\
25.03+4.38\end{array}$ & $37.02 \pm 10.02$ \\
\hline & $\mathbf{T}$ & $34.93 \pm 9.27$ & $33.36 \pm 7.77$ & $33.57 \pm 9.35$ & $36.20 \pm 9.81$ & $37.29 \pm 10.25$ & $34.00 \pm 8.59$ & $34.86 \pm 10.40$ \\
\hline \multirow{2}{*}{ 煦 } & H & $108.23 \pm 25.95$ & $96.78 \pm 22.68$ & $\begin{array}{c}106.44 \pm 32.74 \\
66.98 \pm 16.30\end{array}$ & $\begin{array}{c}112.77 \pm 20.47 \\
61.34 \pm 12.87\end{array}$ & $114.5 \pm 26.18$ & $\begin{array}{l}97.43 \pm 20.25 \\
57.47 \pm 12.13\end{array}$ & $111.3 \pm 18.63$ \\
\hline & $\mathrm{T}$ & $91.72 \pm 31.31$ & $85.62 \pm 24.64$ & $87.53 \pm 32.73$ & $96.48 \pm 30.28$ & $98.40 \pm 35.20$ & $84.11 \pm 26.09$ & $104.5 \pm 23.56$ \\
\hline \multirow{3}{*}{$\frac{0}{0}$} & H & $132.40 \pm 31.83$ & $122.9 \pm 38.95$ & $130.51 \pm 37.18$ & $139.94 \pm 27.76$ & $134.0 \pm 31.86$ & $129.8 \pm 21.76$ & $135.5 \pm 19.43$ \\
\hline & M & $83.76 \pm 27.95$ & $84.30 \pm 25.24$ & $86.11 \pm 25.78$ & $80.71 \pm 24.95$ & $82.44 \pm 37.38$ & $74.68 \pm 28.26$ & \\
\hline & $\mathbf{T}$ & $114.31 \pm 9.21$ & $106.3 \pm 38.54$ & $109.2 \pm 39.05$ & $121.1 \pm 38.53$ & $118.4 \pm 40.97$ & $111.4 \pm 35.47$ & $127.7 \pm 31.55$ \\
\hline
\end{tabular}

Nota: DMD (dinamometría mano derecha), DMI (dinamometría mano izquierda), DEsp (dinamometría espalda), DPie (dinamometría piernas).

En la tabla 3, se presentan los valores obtenidos por décadas de edad y sexo en relación a las variables antropométricas.

Tabla 3: Datos obtenidos de la muestra a estudio expresados por décadas y sexo declarado en relación a las variables antropométricas.

$\begin{array}{lllllll}\text { Total (491) } & 18 / 19 & 20 / 29 & 30 / 39 & 40 / 49 & 50 / 59 & 60 / 68\end{array}$




\begin{tabular}{|c|c|c|c|c|c|c|c|c|}
\hline \multirow{6}{*}{$\begin{array}{l}\text { O } \\
\text { ¿ }\end{array}$} & & $\begin{array}{l}H(n=289) \\
M(n=202)\end{array}$ & $\begin{array}{l}H(n=28) \\
M(n=24)\end{array}$ & $\begin{array}{l}H(n=76) \\
M(n=84)\end{array}$ & $\begin{array}{l}H(n=63) \\
M(n=37)\end{array}$ & $\begin{array}{l}H(n=78) \\
M(n=23)\end{array}$ & $\begin{array}{l}H(n=34) \\
M(n=30)\end{array}$ & $\begin{array}{l}H(n=9) \\
M(n=4)\end{array}$ \\
\hline & & media $\pm \mathrm{DS}$ & media $\pm \mathrm{DS}$ & media $\pm \mathrm{DS}$ & media $\pm \mathrm{DS}$ & media $\pm \mathrm{DS}$ & media $\pm \mathrm{DS}$ & media $\pm \mathrm{DS}$ \\
\hline & $\mathbf{H}$ & $76.63 \pm 10.88$ & $73.03 \pm 10.61$ & $73.12 \pm 9.46$ & $75.50 \pm 10.68$ & $80.88 \pm 10.93$ & $79.57 \pm 10.5$ & $81.80 \pm 20.3$ \\
\hline & $\mathbf{M}$ & $61.10 \pm 9.75$ & $65.52 \pm 11.46$ & $58.84 \pm 8.35$ & $59.23 \pm 5.30$ & $62.31 \pm 13.68$ & 1 & 2 \\
\hline & $\mathbf{T}$ & $70.32 \pm 12.92$ & $69.81 \pm 11.46$ & $66.27 \pm 11.43$ & $70.35 \pm 12.00$ & $75.28 \pm 14.53$ & $60.14 \pm 9.61$ & \\
\hline & & & & & & & $\begin{array}{c}73.10 \pm 13.7 \\
1\end{array}$ & $\begin{array}{c}77.10 \pm 21.5 \\
1\end{array}$ \\
\hline \multirow{4}{*}{$\frac{\pi}{\bar{\sigma}}$} & $\mathbf{H}$ & $175.59 \pm 6.87$ & $177.85 \pm 6.40$ & $176.25 \pm 6.51$ & $176.32 \pm 7.51$ & $175.97 \pm 6.46$ & $172.13 \pm 6.9$ & $169.80 \pm 3.9$ \\
\hline & $\mathbf{M}$ & $163.65 \pm 7.18$ & $171.74 \pm 8.82$ & $163.84 \pm 6.57$ & $163.03 \pm 4.91$ & $161.29 \pm 7.15$ & 1 & 6 \\
\hline & $\mathbf{T}$ & $170.72 \pm 9.10$ & $175.23 \pm 8.02$ & $170.31 \pm 9.01$ & $172.12 \pm 9.19$ & $171.54 \pm 9.48$ & $158.90 \pm 4.7$ & \\
\hline & & & & & & & $\begin{array}{c}2 \\
167.72 \pm 8.8 \\
5\end{array}$ & $\begin{array}{c}167.33 \pm 7.0 \\
0\end{array}$ \\
\hline \multirow{4}{*}{$\frac{5}{0}$} & $\mathbf{H}$ & $79.78 \pm 12.14$ & $82.24 \pm 9.16$ & $77.03 \pm 9.10$ & $80.22 \pm 11.13$ & $78.46 \pm 10.50$ & $78.76 \pm 12.3$ & $84.40 \pm 4.39$ \\
\hline & M & $80.71 \pm 10.67$ & $80.86 \pm 12.01$ & $81.02 \pm 10.95$ & $84.38 \pm 10.70$ & $82.03 \pm 10.78$ & & \\
\hline & $\mathbf{T}$ & $80.19 \pm 11.57$ & $81.65 \pm 10.33$ & $78.94 \pm 10.18$ & $81.54 \pm 11.08$ & $79.54 \pm 10.63$ & $80.31 \pm 9.42$ & $85.75 \pm 5.13$ \\
\hline & & & & & & & $\begin{array}{c}79.28 \pm 11.3 \\
0\end{array}$ & \\
\hline \multirow{4}{*}{ ర్ల్ర } & $\mathbf{H}$ & $97.01 \pm 7.27$ & $98.21 \pm 4.54$ & $95.53 \pm 5.90$ & $96.68 \pm 7.35$ & $96.46 \pm 7.81$ & $97.00 \pm 6.76$ & $103.24 \pm 5.3$ \\
\hline & M & $98.04 \pm 7.25$ & $96.69 \pm 11.09$ & $98.21 \pm 6.58$ & $101.40 \pm 7.44$ & $99.17 \pm 7.26$ & $94.77 \pm 9.91$ & 3 \\
\hline & $\mathbf{T}$ & $97.47 \pm 7.31$ & $97.56 \pm 7.92$ & $96.85 \pm 6.35$ & $98.17 \pm 7.65$ & $97.28 \pm 7.69$ & $96.25 \pm 7.86$ & \\
\hline & & & & & & & & $\begin{array}{c}103.36 \pm 4.7 \\
8\end{array}$ \\
\hline \multirow{3}{*}{ U } & $\mathbf{H}$ & $.82 \pm .11$ & $.83 \pm .07$ & $.80 \pm .07$ & $.82 \pm .08$ & $.81 \pm .12$ & $.81 \pm .14$ & $.81 \pm .06$ \\
\hline & $\mathbf{M}$ & $.82 \pm .10$ & $.84 \pm .14$ & $.82 \pm .08$ & $.83 \pm .09$ & $.83 \pm .13$ & $.86 \pm .18$ & \\
\hline & $\mathbf{T}$ & $.82 \pm .10$ & $.83 \pm .10$ & $.81 \pm .07$ & $.83 \pm .09$ & $.82 \pm .12$ & $.83 \pm .15$ & $.83 \pm .06$ \\
\hline \multirow{3}{*}{ 商 } & H & $5.65 \pm 0.48$ & $5.51 \pm .29$ & $5.48 \pm .33$ & $5.56 \pm .43$ & $5.79 \pm .42$ & $5.90 \pm .37$ & $5.92 \pm .54$ \\
\hline & $\mathbf{M}$ & $5.02 \pm 0.53$ & $5.08 \pm .67$ & $4.92 \pm .32$ & $4.96 \pm .28$ & $5.27 \pm 1.25$ & $5.05 \pm .48$ & \\
\hline & $\mathbf{T}$ & $5.39 \pm 0.59$ & $5.32 \pm .53$ & $5.21 \pm .43$ & $5.37 \pm .48$ & $5.64 \pm .80$ & $5.61 \pm .57$ & $5.80 \pm .56$ \\
\hline \multirow{3}{*}{ 产 } & $\mathbf{H}$ & $6.78 \pm .57$ & $6.71 \pm .65$ & $6.70 \pm .43$ & $6.68 \pm .48$ & $6.93 \pm .58$ & $6.80 \pm .59$ & $7.12 \pm .39$ \\
\hline & M & $5.98 \pm .55$ & $6.06 \pm .61$ & & 5.91 & 6 & $6.07 \pm .71$ & \\
\hline & $\mathbf{T}$ & $6.45 \pm .69$ & $6.43 \pm .70$ & $6.31 \pm .62$ & $6.44 \pm .58$ & $6.59 \pm .75$ & $6.56 \pm .71$ & $6.96 \pm .51$ \\
\hline \multirow{3}{*}{ 商 } & $\mathbf{H}$ & $9.74 \pm .67$ & $9.74 \pm .72$ & $9.58 \pm .58$ & $9.61 \pm .80$ & $9.92 \pm .53$ & $10.01 \pm .60$ & $10.06 \pm .68$ \\
\hline & $\mathbf{M}$ & $9.00 \pm .75$ & $9.12 \pm .84$ & $8.80 \pm .59$ & $8.91 \pm .49$ & $8.85 \pm 1.06$ & $9.10 \pm .77$ & \\
\hline & $T$ & $9.44 \pm .79$ & $9.47 \pm .82$ & $9.21 \pm .70$ & $9.39 \pm .78$ & $9.60 \pm .88$ & $9.71 \pm .78$ & $9.83 \pm .82$ \\
\hline \multirow{3}{*}{$\begin{array}{l}0 \\
\stackrel{m}{\alpha}\end{array}$} & $\mathbf{H}$ & $32.89 \pm 2.86$ & $32.30 \pm 2.96$ & $32.96 \pm 3.00$ & $32.62 \pm 2.66$ & $33.55 \pm 3.45$ & $33.10 \pm 1.99$ & $34.58 \pm 5.71$ \\
\hline & M & $27.93 \pm 2.97$ & $28.28 \pm 2.87$ & $27.00 \pm 2.45$ & $27.58 \pm 2.39$ & $28.30 \pm 3.65$ & $28.54 \pm 3.31$ & \\
\hline & $\mathbf{T}$ & $30.85 \pm 3.79$ & $30.58 \pm 3.51$ & $30.10 \pm 4.05$ & $31.03 \pm 3.48$ & $31.97 \pm 4.24$ & $31.58 \pm 3.28$ & $33.15 \pm 6.19$ \\
\hline \multirow{3}{*}{$\sum_{0}$} & $\mathrm{H}$ & $37.70 \pm 2.63$ & $37.36 \pm 2.62$ & $37.12 \pm 1.66$ & $37.00 \pm 3.12$ & $38.85 \pm 2.93$ & $38.08 \pm 2.45$ & $38.46 \pm 4.73$ \\
\hline & M & $36.08 \pm 5.53$ & $40.67 \pm 17.56$ & $35.31 \pm 2.41$ & $35.24 \pm 1.66$ & $35.76 \pm 2.96$ & $36.40 \pm 4.27$ & \\
\hline & $\mathbf{T}$ & $37.03 \pm 4.15$ & $38.78 \pm 11.56$ & $36.25 \pm 2.24$ & $36.44 \pm 2.85$ & $37.92 \pm 3.24$ & $37.52 \pm 3.20$ & $37.38 \pm 4.98$ \\
\hline \multirow{3}{*}{ : } & H & $13.01 \pm 5.52$ & $11.88 \pm 5.50$ & $12.34 \pm 5.96$ & $12.42 \pm 5.11$ & $13.54 \pm 5.45$ & $14.80 \pm 6.65$ & $17.84 \pm 6.70$ \\
\hline & $\mathbf{M}$ & $19.68 \pm 6.54$ & $16.58 \pm 5.61$ & $17.35 \pm 5.42$ & $20.28 \pm 7.19$ & $20.50 \pm 6.17$ & $22.67 \pm 7.06$ & \\
\hline & $\mathbf{T}$ & $15.75 \pm 6.80$ & $13.89 \pm 5.95$ & $14.74 \pm 6.21$ & $14.91 \pm 6.86$ & $15.64 \pm 6.48$ & $17.43 \pm 7.67$ & $16.90 \pm 6.42$ \\
\hline \multirow{4}{*}{$\begin{array}{l}\text { 옴 } \\
\text { ฉ }\end{array}$} & $\mathbf{H}$ & $15.25 \pm 7.11$ & $12.08 \pm 8.02$ & $12.91 \pm 6.56$ & $13.69 \pm 6.15$ & $18.15 \pm 7.83$ & $20.43 \pm 6.82$ & $19.14 \pm 10.0$ \\
\hline & M & $14.18 \pm 6.64$ & $12.10 \pm 4.16$ & $11.98 \pm 4.28$ & $15.40 \pm 7.52$ & $15.30 \pm 6.30$ & $16.13 \pm 8.30$ & 7 \\
\hline & $\mathbf{T}$ & $14.81 \pm 6.93$ & $12.08 \pm 6.56$ & $12.46 \pm 5.78$ & $14.23 \pm 6.59$ & $17.29 \pm 7.47$ & $19.00 \pm 7.50$ & \\
\hline & & & & & & & & $17.81 \pm 9.58$ \\
\hline \multirow{3}{*}{ क्र } & $\mathbf{H}$ & $13.27 \pm 6.99$ & $11.14 \pm 8.51$ & $11.82 \pm 8.07$ & $11.86 \pm 5.16$ & $15.02 \pm 7.39$ & $14.73 \pm 5.80$ & $16.74 \pm 9.11$ \\
\hline & $\mathbf{M}$ & $13.61 \pm 6.44$ & $12.22 \pm 4.75$ & $12.04 \pm 4.78$ & $13.37 \pm 7.12$ & $13.93 \pm 5.99$ & $13.89 \pm 5.92$ & \\
\hline & $\mathbf{T}$ & $13.41 \pm 6.77$ & $11.60 \pm 7.08$ & $11.92 \pm 6.66$ & $12.34 \pm 5.83$ & $14.69 \pm 6.97$ & $14.45 \pm 5.76$ & $15.51 \pm 8.68$ \\
\hline \multirow{3}{*}{ 움 } & $\mathbf{H}$ & $23.01 \pm 9.52$ & $16.72 \pm 9.73$ & $19.86 \pm 10.46$ & $20.15 \pm 7.39$ & $26.27 \pm 8.08$ & $29.65 \pm 8.87$ & $29.78 \pm 5.41$ \\
\hline & M & $20.64 \pm 7.41$ & $18.81 \pm 7.60$ & $18.53 \pm 6.81$ & $20.45 \pm 7.01$ & $21.33 \pm 7.00$ & $22.20 \pm 8.26$ & \\
\hline & $\mathbf{T}$ & $22.04 \pm 8.79$ & $17.61 \pm 8.82$ & $19.22 \pm 8.88$ & $20.24 \pm 7.21$ & $24.78 \pm 8.05$ & $27.17 \pm 9.26$ & $27.78 \pm 6.88$ \\
\hline \multirow{3}{*}{ 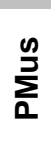 } & $\mathbf{H}$ & $15.91 \pm 7.24$ & $16.96 \pm 8.84$ & $15.38 \pm 6.97$ & $15.28 \pm 6.89$ & $17.12 \pm 7.55$ & $15.38 \pm 7.22$ & $20.96 \pm 5.49$ \\
\hline & $\mathbf{M}$ & $26.68 \pm 8.04$ & $25.12 \pm 9.50$ & $24.93 \pm 7.28$ & $26.84 \pm 7.27$ & $25.73 \pm 7.71$ & $30.92 \pm 9.15$ & \\
\hline & $\mathbf{T}$ & $20.34 \pm 9.24$ & $20.46 \pm 9.88$ & $19.96 \pm 8.55$ & $18.94 \pm 8.82$ & $19.71 \pm 8.52$ & $\begin{array}{c}20.56 \pm 10.7 \\
5\end{array}$ & $20.96 \pm 4.91$ \\
\hline \multirow{3}{*}{$\frac{0}{\alpha}$} & H & $9.64 \pm 4.87$ & $9.37 \pm 5.18$ & $8.96 \pm 4.56$ & $9.31 \pm 4.94$ & $10.63 \pm 5.98$ & $10.78 \pm 6.49$ & $12.46 \pm 4.17$ \\
\hline & M & $16.68 \pm 6.32$ & $15.35 \pm 6.61$ & $15.31 \pm 6.08$ & $16.57 \pm 6.51$ & $15.93 \pm 5.18$ & $17.22 \pm 5.93$ & \\
\hline & $\mathbf{T}$ & $12.53 \pm 6.51$ & $11.93 \pm 6.48$ & $12.00 \pm 6.20$ & $11.61 \pm 6.41$ & $12.23 \pm 6.21$ & $12.93 \pm 6.94$ & $12.81 \pm 3.83$ \\
\hline
\end{tabular}

Nota: peso, talla, CIN (cintura), CAD (cadera), ICC (índice cadera-cintura), DBies (diámetro biestiloideo), DBiep (diámetro biepicondilar) y DBico (diámetro bicondíleo), PBC (perímetro brazo contraído), PPM (perímetro de pierna máxima), PTri (pliegue del tríceps), PSub (pliegue subescapular), PSup (pliegue suprailiaco), PAbd (pliegue abdominal), PMus (pliegue muslo anterior), pierna medial, PPie (pliegue pierna medial). 
Entre las variables analizadas con una mayor correlación con la flexibilidad se encuentran: el pliegue abdominal $(r=-.313)$ y el peso $(r=-.297)$.

Se realiza una prueba $t$ de muestras independientes entre las diferentes variables. En la misma, el resultado obtenido señala que existen diferencias significativas en todas las variables entre hombres y mujeres, a excepción de las siguientes: el pliegue subescapular y el pliegue suprailiaco.

Calculada la ecuación de predicción del SRT en la muestra general mediante una regresión lineal por pasos, las variables que resultan determinantes son: el pliegue abdominal, diámetro biepicondilar y pliegue del tríceps. Sugiere una predicción moderada del $39.1 \%\left(R^{2}=0.391\right)$ en relación al valor de flexibilidad mediante la fórmula:

Flexibilidad $=48.301-.361 \times$ pliegue abdominal $-2.262 \times$ diámetro biepicondilar $+.179 \times$ pliegue del tríceps.

En el cuadro de practicantes de AFD con sexo hombre realizamos una correlación para determinar la relación existente entre las distintas variables y la flexibilidad. Las variables con una mayor correlación fueron: pliegue subescapular $(r=-.262)$, pliegue suprailiaco $(r=-.287)$ y pliegue abdominal $(r=-$ .306). Establecida la ecuación de predicción del SRT, el pliegue abdominal mostró una relación significativa. Sugiere una predicción moderada del $30.6 \%$ $\left(R^{2}=0.306\right)$ en relación al valor de flexibilidad mediante la fórmula:

Flexibilidad $=32.838-.284 \times$ pliegue abdominal.

En el registro de practicantes de AFD con sexo mujer también se realizó una correlación para determinar la relación existente entre las distintas variables y la flexibilidad. Las variables con una mayor correlación son: peso $(r=-.236)$, pliegue subescapular $(r=-.255)$, pliegue suprailiaco $(r=-.238)$, pliegue abdominal $(r=-$ .282) y pliegue pierna medial $(r=-.259)$. Formalizada la ecuación de predicción del SRT, el pliegue abdominal y talla mostraron una relación significativa. Sugiere una predicción moderada del $32.5 \%\left(R^{2}=0.325\right)$ en relación al valor de flexibilidad mediante la fórmula:

Flexibilidad $=71.200-.335 \times$ pliegue abdominal $-.199 \times$ talla.

\section{DISCUSIÓN}

Nuestro interés radica en determinar qué factores miden realmente el SRT, es decir, si las proporciones, composición corporal o la fuerza del sujeto, tienen influencia en los resultados del mismo. Sin embargo, no se han encontrado estudios de referencia que analicen las relaciones existentes entre la flexibilidad, valorada mediante la prueba de SRT, la dinamometría, las variables antropométricas y la actividad física realizada mediante parámetros establecidos como saludables por la ACSM (25). 
Los pliegues subescapular y suprailiaco aparecen como estadísticamente significativos entre sexos. Podría corresponder al lado dominante del sujeto, ya que una mayor habilidad con esa extremidad podría ocasionar adaptaciones musculo-esqueléticas asimétricas (28). Y con ello estar siendo afectado por la biomecánica del movimiento en la práctica de la AFD, movimiento realizado en las zonas anatómicas adyacentes a los pliegues. Arellano \& Kram (29) inciden que con la finalidad de minimizar el coste energético y mejorar el equilibrio lateral, adaptamos nuestra biomecánica con una anchura estrecha de pasos al caminar y un balanceo lateral en los brazos.

Según establece la fórmula general de la muestra, el pliegue del tríceps, el diámetro biepicondilar y el pliegue abdominal aparecen como predictores. Un estudio realizado por Bale et al., (30) concluyó que la diferencia en el porcentaje graso estaba particularmente reflejada en el pliegue del tríceps. Vila et al., (31) distinguen el pliegue del tríceps dentro de su modelo predictivo y relacionado con la composición corporal.

Carrasco et al., (32) muestran una diferencia en atletas que practican deporte con objeto en comparación con otros, en los diámetros biepicondilar, biestiloideo y bicondíleo del brazo. Lermakov et al., (33) destacan mayores diámetros en el brazo y antebrazo en relación a la fuerza desplegada o dinamometría, en deportes de contacto. Norton \& Olds (34) destacan el aumento de los diámetros en atletas que practican deportes de adversario relacionado con nutrición, ergogenia o entrenamiento, lo que se contradice al estudio de Vila et al., (31) donde determina los diámetros como variables antropométricas no modificables por el entrenamiento.

El análisis de regresión lineal determinó el pliegue abdominal como parte de la fórmula predictiva en hombres y el pliegue abdominal y la talla en mujeres.

Encontramos que el valor del pliegue abdominal aumenta significativamente en hombres en la década de los 40/49 años. Y analizando esta variable como una de las variables predictoras de la flexibilidad tanto en hombres como en mujeres, nos resulta una mayor predicción en mujeres $(p=-.335)$ que en hombres $(p=-$ .284). Según Kerr \& Stewart (35) dentro de los factores que afectan a la composición corporal en personas que practican AFD se encuentran: genéticos, crecimiento, envejecimiento y nutrición. El crecimiento y el envejecimiento están acompañados por cambios en el tejido adiposo, tejido muscular y masa ósea. Hrazdíra et al., (36) determinaron que la acumulación de grasa abdominal es un factor limitante en el SRT, cuyo acumulo es mayor en hombres que en mujeres.

La influencia de la talla en el SRT, en relación inversa en mujeres y no en hombres, reafirma los estudios sobre la influencia de las proporciones corporales en el propio test. Nos encontramos con diferencias significativas entre hombres y mujeres $p=.001$. En las mujeres cuanto menor valor de talla mejores resultados en el SRT, lo cual determina que las variables o proporciones longitudinales no influyen en el resultado numérico del SRT. Similares resultados fueron obtenidos por Shephard et al., (37), donde advierten que la talla es un factor que no influye en los resultados del SRT en los hombres, en cambio, la talla de las mujeres es señalada como un factor influyente. 
En la flexibilidad nos encontramos con una menor dispersión de datos en la década de 50/59 años y mayores valores de flexibilidad en sexo femenino. Según Smith \& Miller (8) determinan una alta significatividad entre sexo y el SRT; Mier (17) afirmó que la validez de la flexibilidad isquiotibial fue moderada en mujeres y baja en hombres; sin embargo, Mayorga et al., (18) mostraron que los resultados del SRT tienen una estimación moderada-alta como criterio de validez de la flexibilidad en la musculatura isquiosural, mayor para hombres que para mujeres.

Por lo tanto, la práctica de AFD ocasiona según Kim et al., (38) cambios beneficiosos en la aptitud del músculo esquelético, en la flexibilidad y en la composición corporal; Hrazdíra et al., (36) señalan que las personas activas físicamente presentan mejores resultados de flexibilidad que aquellas inactivas en todos los grupos de edad; Mathunjwa et al., (39) en su estudio con deportistas diferenciados por sexos exponen que sus resultados son mejores según aumenta la edad, pudiendo deberse a adaptaciones debidas al entrenamiento 0 demandas técnicas del deporte específico en cuestión. Matos-Duarte et al., (40) observó que corresponde la mejora de la flexibilidad a su relación con la práctica físico deportiva bajo recomendaciones ACSM.

\section{CONCLUSIONES}

En la muestra total de deportistas encontramos una correlación moderada de diversas variables con los valores de la flexibilidad medida con el SRT, como son: pliegue abdominal y peso.

En la muestra de deportistas hombres las variables que correlacionan con la flexibilidad, medida con el SRT, son: pliegue subescapular, suprailiaco y abdominal y en la muestra de mujeres deportistas se añade la variable peso.

En todas las muestras el nivel de predicción de los valores del SRT hay considerarlos como moderados. Destacar que:

- El pliegue abdominal predice la flexibilidad en la muestra general y en ambos sexos.

- La influencia sobre la flexibilidad, aunque sólo en la muestra general del diámetro biepicondilar y el pliegue del tríceps.

- La talla solo influye en el valor de flexibilidad, en la muestra del sexo femenino.

- La fuerza se considera que no predice los resultados de los valores del SRT.

\section{REFERENCIAS BIBLIOGRAFICAS}

1. Bouchard, C., Shephard, R., Stephens, T., Sutton, J. \& McPherson, B. Exerc fit health: the consens statement. Paper presented at the Exercise, fitness, and health: a consensus of current knowledge: proceedings of the 
International Conference on Exercise, fitness and health, May 29-June 3, 1988, Toronto, Canada.

2. Pate, R. R. The evolving definition of physical fitness. Quest 1988;40(3):174179. https://doi.org/10.1080/00336297.1988.10483898

3. Wells K.F, Dillon E.K. The sit and reach-a test of back and leg flexibility. Res Quart. American Assoc Health Phys Education Recreate 1952;23(1):115-118. https://doi.org/10.1080/10671188.1952.10761965

4. American Alliance for Health, Physical Education, Recreation and Dance (AAHPERD). Health Relat Phys Fittest Man. Reston, Va, USA: AAHPERD; 1980.

5. COUNCIL OF EUROPE. Committee for the Development of Sport: European test of physical fitness. Handb Eurofit Test Phys Fit. Rome: CONI;1988.

6. Pyke, J. Australian Council for Health, Physical Education and Recreation. Australian health and fitness survey. Parkside, South Australia: ACHPER, (1985).

7. Canadian Society for Exercise Physiology. Can Phys Activity Fit Lifestyle Appraisal. Ottawa, ON, Health Canada;1996.

8. Smith, J. F. \& Miller, C. V. The effect of head position on sit and reach performance. Res Q Exercise Sport 1985;56(1):84-85. https://doi.org/10.1080/02701367.1985.10608437.

9. Miñarro, P. A. L., de Baranda Andújar, P. S., García, P. L. R. \& Toro, E. O. A comparison of the spine posture among several sit-and-reach test protocols. J Sci Med Sport 2007;10(6):456-462. https://doi.org/10.1016/i.jsams.2006.10.003 PMid:17298887

10. Hemmatinezhad, M., Afsharnezhad, T., Nateghi, N. \& Damirchi, A. The relationship between limb length with classical and modified back saver sitand-reach tests in student boys. Int J Fit 2009;5(1):69-78.

11. Rubinfeld, M. J. Wygand, J., \& Otto, R. M. Hamstring flexibility as assessed by multiple angle Sit \& Reach box apparatus. Med Sci Sport Exer 2002;34(5):S151. http://dx.doi.org/10.1097/00005768-200205001-00844.

12. Muyor, J. M., Vaquero-Cristóbal, R., Alacid, F. \& López-Miñarro, P. A. Criterion-related validity of sit-and-reach and toe-touch tests as a measure of hamstring extensibility in athletes. J Strength Cond Res 2014;28(2):546-555. https://doi.org/10.1519/JSC.0b013e31829b54fb PMid:24476746

13. Perin, A., Ulbricht, L. \& Borba Neves, E. Contribution of different body segments in Sit and Reach Test. Motricidade 2015;11(2):153-162. http://ds.doi.org/10.6063/motricidade.6006.

14. Simoneau, G. G. The Impact of Various Anthropometric and Flexibility Measurements on the Sit-and-Reach Test. J Strength Cond Res 1998;12(4):232-237. https://doi.org/10.1519/00124278-199811000-00005

15. Chillón, P., Castro-Piñero, J., Ruiz, J. R., Soto, V. M., Carbonell-Baeza, A., Dafos, J. \& Ortega, F. B. Hip flexibility is the main determinant of the backsaver sit-and-reach test in adolescents. J Sport Sci 2010;28(6):641-648. https;//doi.org/10-1080/02640411003606234 PMid:20397092

16. López-Miñarro, P. A. \& Rodríguez-García, P. L. Hamstring muscle extensibility influences the criterion-related validity of sit-and-reach and toetouch tests. J Strength Cond Res 2010;24(4):1013-1018.

https://doi.org/10.1519/JSC.0b013e3181c7c60d PMid:20300025 
17. Mier, C. M. Accuracy and feasibility of video analysis for assessing hamstring flexibility and validity of the sit-and-reach test. Res Q Exercise Sport 2011;82(4):617-623. https://doi.org/10.1080/02701367.2011.10599798 PMid:22276403

18. Mayorga-Vega, D., Merino-Marban, R. \& Viciana, J. Criterion-related validity of sit-and-reach tests for estimating hamstring and lumbar extensibility: A meta-analysis. J Sport Sci Med 2014;13(1):1-14.

https://doi.org/10.4100/jhse.2014.91.18

19. Ayala, F., de Baranda, P. S., Croix, M. D. S. \& Santonja, F. Reproducibility and criterion-related validity of the sit and reach test and toe touch test for estimating hamstring flexibility in recreationally active young adults. Phys Ther Sport 2012;13(4):219-226. https://doi.org/10.1016/j.ptsp.2011.11.001 PMid:23068896.

20. World Health Organization. Expert Committee on Physical Status: The use and Interpretation of Anthropometry. WHO tech rep ser 854. Geneva: WHO, 1995.

21. Kraemer W.J, Adams K, Cafarelli E., Dudley G.A., Dooly C., Feigenbaum M.S., Fleck S.J., Franklin B., Fry A.C., Hoffman J.R. \& al. American College of Sports Medicine Position Stand. Progression models in resistance training for healthy adults. Med Sci Sports Exerc 2002;34(2):364-380. https://doi.org/10.1097/00005768-200202000-00027 PMid:11828249

22. Chandrasekaran, B., Ghosh, A., Prasad, C., Krishnan, K. \& Chandrasharma, B. (2010). Age and anthropometric traits predict handgrip strength in healthy normals. J Hand Microsurg 2010;2(2):58-61. https://doi.org/10.1007/s12593010-0015-6. PMid:22282669 PMCid:PMC3122705

23. Silva, N. d. A., Menezes, T. N. d., Melo, R. L. P. d. \& Pedraza, D. F. Handgrip strength and flexibility and their association with anthropometric variables in the elderly. Rev Assoc Med Bras 2013;59(2):128-135. https://doi.org/10.1016/..ramb.2012.10.002 PMid:23582553

24. Sharma, H. B. \& Kailashiya, J. The Anthropometric Correlates for the Physiological Demand of Strength and Flexibility: A study in Young Indian Field Hockey Players. J Clin Diagn Res 2017;11(6):CC01-CC05. https://doi.org/10.7860/JCDR/2017/26358.9965 PMid:28764148 PMCid:PMC5535341

25. Garber CE, Blissmer B, Deschenes MR. \& al. American College of Sports Medicine Position Stand. Quantity and Quality of Exercise for Developing and Maintaining Cardiorespiratory, Musculoskeletal, and Neuromotor Fitness in Apparently Healthy Adults: Guidance for Prescribing Exercise. Med Sci Sports Exerc 2011;43(7):1334-1359. https://doi.org/10.1249/MSS.0b013e318213fefb PMid:21694556

26. Stewart, A., Marfell-Jones, M., Olds, T. \& de Ridder, H. International standards for anthropometric assessment: ISAK: Lower Hutt. New Zealand, 2011.

27. Hopkins W, Marshall S, Batterham A, Hanin J. Progressive statistics for studies in sports medicine and exercise science. Med Sci Sports Exerc. 2009;41(1):3. https://doi.org/10.1249/MSS.0b013e31818cb278 PMid:19092709

28. Gleim, G. W. \& McHugh, M. P. Flexibility and its effects on sports injury and performance. Sports Med 1997;24(5):289-299. https://doi.org/10.2165/00007256-199724050-00001 PMid:9368275 
29. Arellano, C. J. \& Kram, R. The effects of step width and arm swing on energetic cost and lateral balance during running. $J$ Biomech 2011;44(7):1291-1295. https://doi.org/10.1016/i.jbiomech.2011.01.002 PMid:21316058

30. Bale, P., Rowell, S. \& Colley, E. (1985). Anthropometric and training characteristics of female marathon runners as determinants of distance running performance. J Sport Sci 1985;3(2):115-126. https://doi.org/10.1080/02640418508729741 PMid:4094022

31. Vila, M., Manchado, C., Abraldes, J. \& Ferragut, C. Predicting playing status in professional water polo players: analysis by gender. J Sport Med Phys Fit 2017;58(9):1234-39. DOI: 10.23736/S0022-4707.17.07201-2.

32. Carrasco Páez, L., Martínez Pardo, E. \& Nadal Soler, C. Anthropometric profile, somatotype and body composition of young paddlers. Rev Int Med Cienc AC 2005;5(19):270-282.

33. Iermakov, S., Podrigalo, L. V. \& Jagiełło, W. Hand-grip strength as an indicator for predicting the success in martial arts athletes. Arch Budo 2016;12:179186.

34.Norton, K. \& Olds, T. Morphological evolution of athletes over the 20th century. Sports Med 2001;31(11):763-783. https://doi.org/10.2165/00007256-200131110-00001 PMid:11583103

35. Kerr D. \& Stewart A. Body composition in sport. En: Ackland TR, Elliott BC \& Bloomfield J, editors. Applied anatomy and biomechanics in sport. Chicago, IL: Hum Kinet 2009:67-85.

36. Hrazdíra, E., Grasgruber, P. \& Kalina, T. The comparison of flexibility in the Czech population aged 18-59 years. J Hum Sport Exerc 2013;8(2):S135S140. https://doi.org/10.4100/jhse.2012.8.Proc2.16

37. Shephard, R. J., Berridge, M. \& Montelpare, W. On the generality of the "sit and reach" test: an analysis of flexibility data for an aging population. Res $Q$ Exercise Sport 1990;61(4):326-330.

https://doi.org/10.1080/02701367.1990.10607495 PMid:2132890

38. Kim, H.-B., Stebbins, C. L., Chai, J.-H. \& Song, J.-K. Taekwondo training and fitness in female adolescents. J Sport Sci 2011;29(2):133-138. https://doi.org/10.1080/02640414.2010.525519 PMid:21170802

39. Mathunjwa, M., Mugandani, S., Djarova-Daniels, T., Ngcobo, M. \& Ivanov, S. Physical, anthropometric and physiological profiles of experienced junior male and female South African Taekwondo athletes. Afr J Phys Health Education Recreat Dance 2015;21(4:2):1402-1416.

40. Matos-Duarte, M., Martínez-de-Haro, V., Sanz-Arribas, I., Andrade, A. \& Chagas, M. H. Longitudinal study of functional flexibility in older physically active. Rev Int Med Cienc AC 2017;17(65):121-137. http://dx.doi.org/10.15366/rimcafd2017.65.008.

Número de citas totales / Total references: 40 (100\%)

Número de citas propias de la revista / Journal's own references: 1 (2,5\%)

Rev.int.med.cienc.act.fís.deporte - vol. 20 - número 78 - ISSN: 1577-0354 